\title{
NEUTRON DIAGNOSTICS FOR VOID MONITORING IN BOILING WATER REACTORS
}

\section{Jan Blomgren*}

Department of Neutron Research

Uppsala University

Box 525, S-751 20 Uppsala, Sweden

E-mail: Jan.Blomgrenetsl.uu.se

In present-day Boiling Water Reactors (BWR), the only in-core neutron diagnostics in fullpower operation (Power-Range Mode, PRM) is performed with fission-based ionisation chambers loaded with ${ }^{235} \mathrm{U}$. Since both the neutron flux and the neutron-induced fission cross section on ${ }^{235} \mathrm{U}$ are the highest at thermal energies, the count rate from such PRM detectors is essentially due to the thermal neutrons. It has recently been proposed that adding PRM detectors loaded with ${ }^{238} \mathrm{U}$, thereby being sensitive only to fast neutrons (above about $1.5 \mathrm{MeV}$ ), to the BWR core could facilitate void monitoring.

The neutron energy spectrum in a BWR is very sensitive to the boiling. Since the moderation of neutrons requires the presence of hydrogen, which in reality means water, the moderation gets significantly less efficient in regions of the reactor where the void fraction (the fraction of the water that is in steam phase) is high. Because the density of steam is significantly lower than when water is in liquid phase, increased void results in reduced moderation. Less moderation results in a neutron energy spectrum with a lower fraction of thermal neutrons and a correspondingly larger number of fast neutrons. Thereby, a simultaneous measurement of the fluxes of thermal and fast neutrons may be used to determine the void in a BWR. The potential of this technique will be outlined, as well as applications in realistic technical systems.

International Workshop on Fast Neutron Detectors and Applications

April, 3 - 6, 2006

University of Cape Town, South Africa

\footnotetext{
* Speaker.
} 


\section{Introduction}

In Boiling Water Reactors (BWR), the presently second most common reactor design in the world, the uranium fuel is surrounded by water that is brought to boiling within the reactor core by the heat released due to fission. In the lower portion of the core, only liquid water moderator is present. As the water flows through the fuel bundles from the bottom to the top, an increasing fraction of the flowing water is in the form of steam. BWRs depend upon this void fraction in their operation because of two discrete phenomena. Firstly, the heat conductivity from the fuel rods to the steam is optimal if a thin water layer is maintained at the fuel surfaces, because the two-step heat transfer from fuel rod to water and from water to steam is far more efficient than a single-step transfer from rod to steam directly. Thus, if the thin water film is evaporated, the cooling of the fuels is dramatically reduced, and this dry-out phenomenon can rapidly lead to overheating, with severe fuel damages as result.

Secondly, the energy release depends on the moderation. The neutrons emitted in fission display an energy distribution ranging from about $0.1 \mathrm{MeV}$ up to a few $\mathrm{MeV}$, with a maximum typically in the $0.5-1.0 \mathrm{MeV}$ range. After release, these fast neutrons are moderated, i.e., are slowed down, primarily due to collisions with the hydrogen in the water, resulting in thermal neutrons of very low energy, typically $0.025 \mathrm{eV}$. At any given moment there are both thermal and fast neutrons present, as well as neutrons of intermediate energies. The properties of a BWR depend strongly on the absolute neutron flux, but also on the relative distribution of neutron energies. Since the moderation of neutrons requires the presence of hydrogen, which in reality means water, the moderation gets significantly less efficient in regions of the reactor where the void fraction is high. Less moderation results in a neutron energy spectrum with a lower fraction of thermal neutrons and a correspondingly larger number of fast neutrons. Thereby, a simultaneous measurement of the fluxes of thermal and fast neutrons may be used to determine the void in a BWR.

\section{Neutron monitoring for void determination}

\subsection{Present neutron diagnostics in BWRs}

In most BWRs today, the only neutron diagnostics in full-power operation is fission-based ionisation chambers loaded with ${ }^{235} \mathrm{U}$, so-called Power Range Monitors (PRM). Since neutroninduced fission in ${ }^{235} \mathrm{U}$ is also the main heat production reaction in a BWR, this signal gives a reasonably good measure of the power of the reactor. For an introduction to detector techniques in reactor environments, see, e.g. ref. [1]. A typical PRM detector is based on two cylinders, an inner and an outer one, used as electrodes, with a gas in between, often argon. A bias voltage is applied to the two electrodes. One of the electrodes is coated with ${ }^{235} \mathrm{U}$. When neutrons induce fission in the ${ }^{235} \mathrm{U}$ coating, fission fragments are released into the gas-filled gap, which cause ionization of the gas. Thereby, an electric current can flow between the electrodes. This current, which is proportional to the neutron flux, is transported out of the reactor in a coaxial cable for further processing. The ionization chamber gas is often under overpressure to ensure that the range of the fission fragments does not exceed the gap dimensions. Typical dimensions of these detectors are up to $10 \mathrm{~cm}$ in length, whereof the active volume comprises $2-3 \mathrm{~cm}$ length, and about $2 \mathrm{~cm}$ in diameter. 
In BWRs, the detectors are placed in vertical tubes inside the reactor core. Typically, four detectors are placed in the same tube at different heights (about $1 \mathrm{~m}$ separation), and 30-40 tubes are installed, resulting in about 150 detectors in total. In addition, some detectors may be provided with a motorized drive to allow traverses though the reactor core.

\subsection{Fast neutron detection}

As described above, in present BWRs, fast neutrons are not explicitly monitored. Technically, fast neutron diagnostics can be achieved with various types of devices, for instance ionization chambers. In that case the coating inside, on the inner cylinder, is an element with a neutron energy threshold for fission (like ${ }^{238} \mathrm{U}$ or ${ }^{232} \mathrm{Th}$ ). The performance of these fast-neutron detectors is very similar to the presently used thermal-neutron detectors, and essentially identical readout is used. The fission cross section for ${ }^{238} \mathrm{U}$, the dominating uranium isotope in natural uranium (and in the fuel of a BWR) is very different from the ${ }^{235} \mathrm{U}$ cross section. The cross section for ${ }^{238} \mathrm{U}$ is almost zero below a threshold energy of about $1 \mathrm{MeV}$, while above that energy the cross section is comparable to that of ${ }^{235} \mathrm{U}$. This means that a fission monitor loaded with pure ${ }^{238} \mathrm{U}$ would be sensitive essentially only to fast neutrons.

\subsection{Determination of the void fraction}

The void fraction can be determined from a simultaneous measurement of the thermal and fast neutron flux, since the ratio of these two fluxes depends on the moderation and thereby on the void. Practically, this can be accomplished by measuring the ratio of the currents in two detectors in a detector pair, i.e., where one detector is sensitive to thermal neutrons and the other to fast neutrons.

In a realistic application, the ratio depends on many different technical parameters of the reactor, the fuel and the state of operation. Moreover, they are different in different regions of the reactor, and change over time since the isotopic composition of the fuel changes with burnout. Therefore, the relation between the current ratio and the void fraction has to be computed by large core simulation codes, like POLCA or CASMO, for relevant positions and operational parameters in the reactor.

The results in figure 1 have been obtained in a calculation presuming two detectors, one being the standard neutron-induced fission neutron detector based on ${ }^{235} \mathrm{U}$, and the other being a similar detector loaded with pure ${ }^{238} \mathrm{U}$. Hence, figure 1 shows a realistic simulation of the performance of a realistic system illustrating a computer calculation of the void and the ratio of fast-to-thermal neutrons. The calculation refers to a real core that has been run recently in a commercial BWR. Each datum point in the figure refers to a measurement position along a vertical channel in the reactor, going from the bottom (to the left) to the top (to the right) of the BWR. It can be seen that there is essentially no void at the bottom of the reactor, while the void increases when moving up in the reactor, and so does the fast/thermal neutron ratio. At the top end of the reactor, i.e., above the uranium fuel, this relation is no longer valid, but over essentially the entire volume where fission takes place, it is present. (The fast/thermal ratio has an arbitrary scale.) It should be pointed out, however, that this concept is not limited to the two uranium isotopes ${ }^{235} \mathrm{U}$ and ${ }^{238} \mathrm{U}$. Also other combinations of a fissile nuclide and a nuclide with a fission threshold at a suitable energy could in principle be used. 


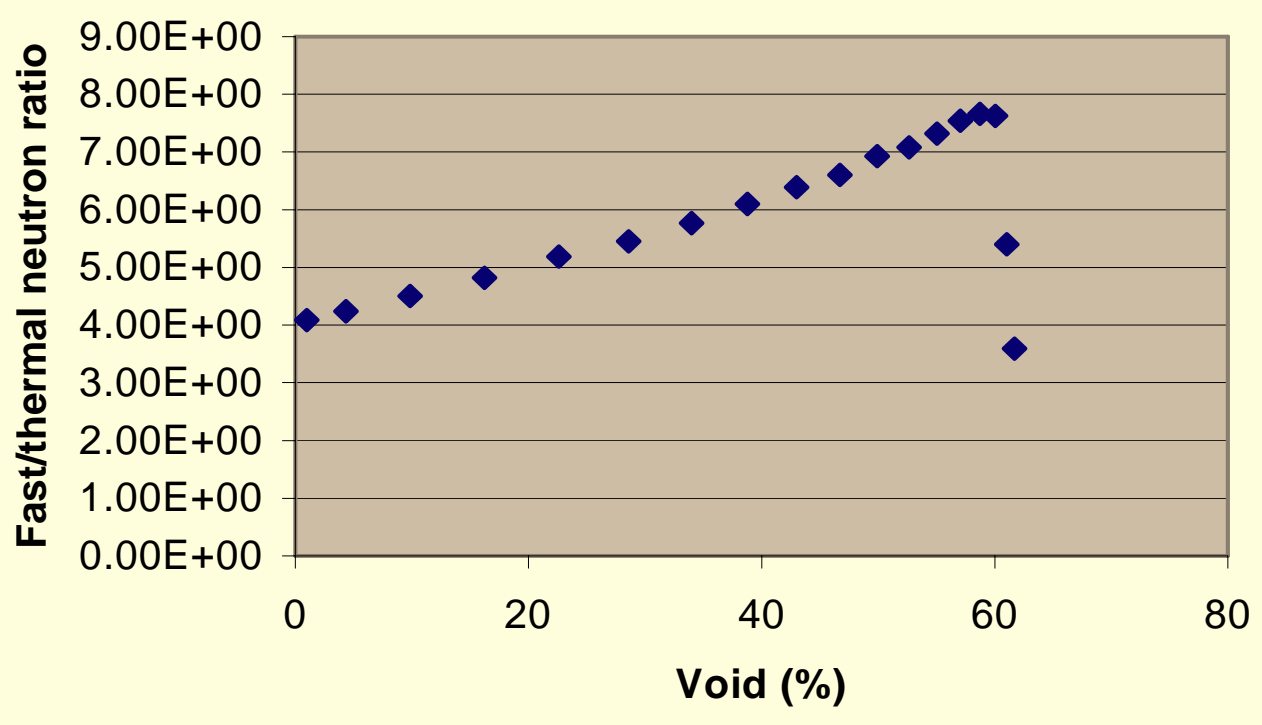

Figure 1: Relation between void and the fast/thermal neutron fraction. The simulation has been performed for a realistic core in a commercially operated reactor. The vertical scale is arbitrary.

\section{Previous research on nuclear void measurement techniques}

\subsection{Why has this not been tried before?}

It could seem like a paradox that such a conceptually simple idea - to combine two detectors, whereof one already existing - should be of any news value. Intuitively, one might assume that this should have already been attempted. There are, however, historic reasons. The standard incore neutron monitors in BWRs are based on ionization chamber techniques. During the design, development and deployment phase of BWR technology (1955-75), many different monitoring schemes were attempted. At that time, however, ionization chambers had not matured to such a level of performance that they could be used for the present application. It was not until BWRs had been established and in regular operation that the ionization chamber technique had reached a sufficiently high level for the present concept to be possible to deploy. At that time, however, the general level of activity on development of genuinely new monitoring concepts had essentially vanished at academic and research institute organizations.

With the recent resurrected interest in academic research on nuclear power applications, there are today possibilities to develop new monitoring concepts for existing LWRs in general and BWRs in particular, as well as Gen-IV type concepts. Below, the present status of knowledge and previous research in nuclear void monitoring techniques for BWRs are outlined.

\subsection{Early nuclear void measurement techniques}

In the early years of LWR development, a number of various concepts for in-core void monitoring were attempted. Reviews of these early R\&D activities can be found in refs. [2, 3, 4, 5]. Below, some of these early investigations are described. 
It was early on observed [6] that the thermal neutron flux surrounding an absorbing rod can be modified by the void, insofar that the flux increases with the void fraction. Based on this, two monitoring techniques were tested in the Halden heavy water reactor [7]. In the first test, the flux was measured using activation of a copper foil. It was concluded that the method as such worked (although the precision was far below what we aim for in the present project), but that the technique would not be very useful in a power reactor. The reason for that is that foils need to be transported in and out of the reactor. Besides the practical problems, they are activated also during the transport, and it takes quite some time to achieve the result of the measurement, i.e., an instantaneous result cannot be obtained. In a second test a uranium thermoelement was used. Also in this case it was concluded that the principle worked, but that the precision was insufficient. The authors did, however, speculate about the possibility to use ionization chambers, which at that time had been used for the first time in a reactor [8].

The basis of the present work is that the fast/thermal neutron flux ratio depends on the void. Such a relation, however weaker, is true also for the epithermal/thermal neutron flux ratio. The epithermal/thermal ratio can be measured relatively easily using the fact that cadmium has a very large absorption cross section up to about $0.5 \mathrm{eV}$, while the cross section drops dramatically above that energy. At Argonne, a system based on this concept was tested in the EBWR reactor [9, 10]. Cobalt wires with and without cadmium wrapping were irradiated in the EBWR core. The induced activity was measured after irradiation, and the activity ratio was used to extract the epithermal/thermal neutron flux ratio, which in turn was used to determine the void. The final result was checked versus computations of the void. There is indeed a correlation between the void and the flux ratio, but the scatter of the data around the calculation is larger than what is acceptable for a practical method. Overall, the method was estimated to have an uncertainty of $15 \%$, and the technique as such (wire activation with post-irradiation analysis) is not well suited for practical industrial implementation. The authors concluded that the method should be applicable also in power-producing reactors, but no subsequent development in that direction has ever been published.

A test was, however, conducted in a test reactor [11], but it did not produce satisfactory results because the radial variation of the cadmium ratio was too large within a subassembly for accurate measurements of the void fraction distribution. These two development projects are representative for a range of similar activities in the early days of BWR development. A more complete listing can be found in ref. [2]. In conclusion, essentially all these concepts were based on activation, and the measurements did in reality concern thermal and epithermal fluxes. It should be emphasized that the fast/thermal flux ratio is much more sensitive to void changes than the epithermal/thermal ratio is.

Direct detection methods were already identified around 1960 to be of paramount importance to make these techniques practical. At that time, no suitable neutron detectors were available. The first steps towards development of the ionization chambers of today had, however, been taken. Below, the development of ionization chambers for reactor use is outlined.

\subsection{Ionization chambers for BWR in-core neutron detection}

As was pointed out above, the first use of ionization chambers in a reactor was reported in 1958 [8]. At that time, one of the major limitations was the very short life span of such a detector. The gradual burn-up of the fissile material in the detector resulted in a rapid and significant effi- 
ciency decrease. This problem was to a large degree remedied by the introduction of regenerative chambers, in which a fertile material is converted into a fissile element at a rate that is close to the burn-up of the original fissile material. The first such detectors, reported around 1975, used ${ }^{239} \mathrm{Pu}$ as original fissile component and ${ }^{238} \mathrm{U}$ as fertile element [12]. Breeding of ${ }^{238} \mathrm{U}$ into ${ }^{239} \mathrm{Pu}$ resulted in a sensitivity change of less than $5 \%$ for an integrated fluence that would have resulted in a $50 \%$ change using ${ }^{239} \mathrm{Pu}$ alone. Soon thereafter, detectors with mixtures of ${ }^{234} \mathrm{U}$ and ${ }^{235} \mathrm{U}$ were developed, and this is by far the most common design today.

\section{Outlook}

The principles outlined in the present article constitute the basis for a research project that has recently been initiated. In a first phase, detailed simulations of how the neutron spectrum depends on a large number of conditions, like void, fuel composition, control rods and location in the reactor, will be performed. In a second phase, the results of these simulations will be used to guide the design of a prototype system to be experimentally tested in a research reactor, where the void and neutron spectrum can be controlled, and measured also by other instruments. It has already been established that the principle as such works, but research and technical development is needed to assess the uncertainty to which the void can be determined in a practical implementation.

\section{Acknowledgments}

The collaboration with Fredrik Winge is gratefully acknowledged. This work was financially supported by the Swedish Nuclear Fuel and Waste Management Company, the Swedish Nuclear Power Inspectorate, Ringhals AB, Forsmarks Kraftgrupp AB, the Swedish Defense Research Agency, the Swedish Nuclear Safety and Training Centre, and the European Union.

\section{References}

[1] G.F. Knoll, Radiation Detection and Measurement, Wiley \& Sons, New York, 1979.

[2] J.F. Boland, Nuclear Reactor Instrumentation (In-core), Gordon \& Breach, New York, 1970.

[3] J.M. Harrer and J.G. Beckerley, Nuclear Power Reactor Instrumentation Systems Handbook, vol. 1, TID-25952-P1, 1973.

[4] J.M. Harrer and J.G. Beckerley, Nuclear Power Reactor Instrumentation Systems Handbook, vol. 2, TID-25952-P2, 1974.

[5] Proceedings of the Specialists Meeting on In-Core Instrumentation and Reactor Assessment, Nuclear Energy Agency (NEA), OECD, 1984.

[6] J.M. Døderlein, The Influence of Airgaps on the Neutron Flux Around Cylindrical Absorbing Rods in a Moderating Medium, Proceedings of the Second International Conference on the Peaceful Uses of Atomic Energy, Vol. 13, P/570, United Nations, Geneva, 1958.

[7] H. Ager-Hanssen and J.M. Døderlein, A Method for Measuring Steam Voids in Boiling Water Reactors, Proceedings of the Second International Conference on the Peaceful Uses of Atomic Energy, vol. 11, p. 463, United Nations, Geneva, 1958.

[8] A.L. Gray, Nuclear Power, p. 172, 1958. 
[9] J.A. Thie, and J. Biedelman, Demonstration of the UTB Voidmeter, Test report no. 72A, in EBWR Test Reports, ed. V.M. Kolba, ANL-6229, 1960.

[10] J.A. Thie, J. Biedelman and B. Hoglund, Void Measurement in a Boiling Reactor, Nucl. Sci. Eng., vol. 11, p. 1, 1961.

[11] BORAX-V Project Staff, Experiments with Central Superheater Core CSH-1, BORAX-V, USAEC Report ANL-6961, Argonne National Laboratory, January 1965, p. 65.

[12] H. Böck and E. Balcar, Nucl. Instr. Meth., vol. 124, p. 563, 1975. 